PROCEEDINGS OF THE

AMERICAN MATHEMATICAL SOCIETY

Volume 134, Number 7, Pages 1917-1925

S 0002-9939(05)08188-8

Article electronically published on December 16, 2005

\title{
ON INVARIANT DISTANCES ON ASYMPTOTIC TEICHMÜLLER SPACES
}

\author{
HIDEKI MIYACHI
}

(Communicated by Juha M. Heinonen)

\begin{abstract}
In this paper, we will establish that any invariant distance on asymptotic Teichmüller space is a complete distance.
\end{abstract}

\section{INTRODUCTION}

By an invariant distance on a complex Banach manifold $X$, we mean a pseudodistance which satisfies the distance decreasing property for every holomorphic functions between $X$ and the unit disc $\mathbb{D}$ in $\mathbb{C}$ (cf. 33.1). Invariant distances are powerful tools for studying the analytic structures of complex Banach manifolds (cf. [3] and 9]) and are also important objects of research in Teichmüller theory (see [6] and [10]). The Carathéodory distance and the Kobayashi distance are typical examples of invariant distances.

In [5, C. Earle, F. Gardiner and N. Lakic showed that every asymptotic Teichmüller space admits a structure of a complex Banach manifold. The aim of this paper is to show that any invariant distance is complete on asymptotic Teichmüller space. Namely, we will show

Theorem 1. For every Riemann surface $R$, any invariant distance on $A T(R)$ is a complete distance.

Recently, C. Earle, V. Markovic and D. Saric obtained that $A T(R)$ is embedded in a Banach space as a bounded domain (cf. \$2.4). Therefore, the topology inherited from the Carathéodory distance $c_{A T(R)}$ coincides with that from the Banach-manifold structure (cf. Theorem IV.2.2 of [9]). In addition, we will obtain

Theorem 2. There exist universal constants $D_{1}$ and $D_{2}$ with the following property: Let $d$ be an invariant distance on $A T(R)$. Then

$$
c_{A T(R)}\left(p_{1}, p_{2}\right) \leq d\left(p_{1}, p_{2}\right) \leq D_{1} c_{A T(R)}\left(p_{1}, p_{2}\right)
$$

for all $p_{1}, p_{2} \in A T(R)$ with $c_{A T(R)}\left(p_{1}, p_{2}\right) \leq D_{2}$.

Thus, the topology inherited from any invariant distance is locally biLipschitz equivalent to that from $c_{A T(R)}$.

In the case when given invariant distance is inner, the distance is globally biLipschitz equivalent to the Kobayashi distance: Recall that an inner distance is a pseudodistance defined from the lengths of rectifiable paths connecting two points

Received by the editors July 16, 2004 and, in revised form, February 1, 2005.

2000 Mathematics Subject Classification. Primary 32G15, 30F25, 30F60.

(C)2005 American Mathematical Society Reverts to public domain 28 years from publication 
(cf. 33.2 see also p. 128 of [9]). Theorem 2 asserts that a rectifiable path for the Kobayashi distance is also a rectifiable path for any invariant distance and vice versa, and these lengths are comparable (cf. Proposition 3.1). Since the Kobayashi distance is inner (cf. Corollary V.4.2 of [9]), we conclude

Corollary 1. Any inner invariant distance on $A T(R)$ is biLipschitz equivalent to the Kobayashi distance on $A T(R)$ with biLipschitz constants universal.

As an application of our theorems, we obtain information of the complex analytic structures of asymptotic Teichmüller spaces: A domain $X$ in a Banach space $B$ is said to be a domain of holomorphy (resp. an $H^{\infty}$-domain of holomorphy) if there is no domain $X^{\prime} \subset B$ containing $X$ such that any holomorphic function (resp. bounded holomorphic function) on $X$ is extended to $X^{\prime}$. It is known that any bounded domain in a Banach space whose Carathéodory distance is complete is an $H^{\infty}$-domain of holomorphy, and hence it is a domain of holomorphy (cf. Proposition 5.5 of [3]). Combining our Theorem 1 and Earle-Markovic-Saric's embedding theorem, we deduce

Corollary 2. For every Riemann surface $R, A T(R)$ is biholomorphically equivalent to an $H^{\infty}$-domain of holomorphy.

Remark 1. The method in this paper also works for the Teichmüller space $T(R)$ and the Teichmüller space $T_{0}(R)$ of asymptotically conformal mappings for every Riemann surface $R$ (cf. [4] and [5]).

Convention. Since $A T(R)$ consists of one point when $R$ is analytically finite, we concentrate only on hyperbolic Riemann surfaces throughout this paper.

\section{Notation}

2.1. Quasiconformal isotopies. Let $R$ be a Riemann surface. Let $\Gamma$ be the Fuchsian group acting on $\mathbb{D}$ with $\mathbb{D} / \Gamma=R$ and denote by $\Lambda(\Gamma)$ the limit set of $\Gamma$. Then $\bar{R}=(\overline{\mathbb{D}}-\Lambda(\Gamma)) / \Gamma$ is an orbifold with interior $R$ and boundary $(\partial \mathbb{D}-\Lambda(\Gamma)) / \Gamma$. We say that $(\partial \mathbb{D}-\Lambda(\Gamma)) / \Gamma$ is the ideal boundary of $R$, and denote it by $\partial^{i d} R$.

Let $R$ and $S$ be Riemann surfaces. Two quasiconformal mappings $f$ and $g$ from $R$ to $S$ are said to be quasiconformally isotopic rel $\partial^{i d} R$ if there exist a constant $K \geq 1$ and a continuous family $\left\{g_{t}\right\}_{t \in[0,1]}$ of $K$-quasiconformal mappings such that $g_{0}=f, g_{1}=g$ and $g_{t}(p)=f(p)=g(p)$ for $(p, t) \in \partial^{i d} R \times[0,1]$.

2.2. Differentials vanishing at infinity. Let $R$ be a Riemann surface and let $\Gamma$ be a Kleinian group acting on a domain $D \subset \widehat{\mathbb{C}}$ with $D / \Gamma=R$. Let $L^{\infty}(D, \Gamma)$ denote the Banach space of $L^{\infty}$-functions $\mu$ on $D$ satisfying the compatibility condition $\mu \circ \gamma \cdot\left(\overline{\gamma^{\prime}} / \gamma^{\prime}\right)=\mu$ for all $\gamma \in \Gamma$. This compatibility condition allows us to identify the space $L^{\infty}(D, \Gamma)$ with the space $L^{\infty}(R)$ of bounded measurable $(-1,1)$ forms on $R$. In particular, the absolute value $|\mu|$ is recognized as a measurable function on $R$. We denote by $\|\cdot\|_{\infty}$ the essential supremum norm on $L^{\infty}(R)$.

We say that $\mu \in L^{\infty}(R)\left(\cong L^{\infty}(D, \Gamma)\right)$ vanishes at infinity on $R$ when for any $\epsilon>0$, there is a compact set $C \subset R$ such that $|\mu|<\epsilon$ a.e. on $R-C$. We denote by $L_{0}^{\infty}(R)\left(\right.$ resp. $\left.L_{0}^{\infty}(D, \Gamma)\right)$ the closed subspace of $L^{\infty}(R)\left(\right.$ resp. $\left.L^{\infty}(D, \Gamma)\right)$ consisting of all $\mu \in L^{\infty}(R)$ vanishing at infinity. A quasiconformal mapping $f$ on $R$ is said to be asymptotically conformal if its complex dilatation vanishes at infinity. We set 
$\widehat{L}(R):=L^{\infty}(R) / L_{0}^{\infty}(R)$. Denote by $[\mu]_{\hat{L}}$ the equivalence class of $\mu \in L^{\infty}(R)$ in $\widehat{L}(R)$ and by $\|\cdot\|_{\hat{L}}$ the quotient norm.

Let $B_{2}(D, \Gamma)$ be a complex Banach space of holomorphic functions $\phi$ on $D$ with $\sup \left\{\lambda_{D}(z)^{-2}|\phi(z)|, z \in D\right\}<\infty$ and satisfy the compatibility condition $\phi \circ \gamma$. $\left(\gamma^{\prime}\right)^{2}=\phi$ for all $\gamma \in \Gamma$, where $\lambda_{D}$ is the hyperbolic metric on $D$. We say that $\phi \in B_{2}(D, \Gamma)$ vanishes at infinity if the associating $(-1,1)$-form $\lambda_{D}^{-2} \bar{\phi}$ vanishes at infinity on $R$, and denote by $B_{2}^{0}(D, \Gamma)$ the Banach space consisting of all $\phi \in$ $B_{2}(D, \Gamma)$ vanishing at infinity. Set $\widehat{B}(\Gamma):=B_{2}\left(\mathbb{D}^{-}, \Gamma\right) / B_{2}^{0}\left(\mathbb{D}^{-}, \Gamma\right)$.

2.3. Asymptotic Teichmüller spaces. Let $R$ be a Riemann surface. The asymptotic Teichmüller space $A T(R)$ of $R$ is, by definition, the space of the equivalence classes of quasiconformal mappings $f$ from $R$ onto a variable Riemann surface $f(R)$. Two mappings $f$ from $R$ to $R_{0}$ and $g$ from $R$ to $R_{1}$ are equivalent if there is an asymptotically conformal mapping $h: R_{0} \rightarrow R_{1}$ such that $h \circ f$ and $g$ are quasiconformally isotopic rel $\partial^{i d} R$. We denote by $[f]_{A T}$ the equivalence class of $f$ in $A T(R)$. It is known that $A T(R)$ admits the natural structure of a complex Banach manifold (cf. [5]). The Teichmüller space $T(R)$ of $R$ has the same definition with one exception. The mapping $h$ has to be conformal. Since conformal mappings are asymptotically conformal, there is a canonical projection $T(R) \rightarrow A T(R)$.

2.4. Bers embeddings and asymptotic Bers maps. Let $R$ be a Riemann surface and let $\Gamma$ be the Fuchsian group acting on $\mathbb{D}$ uniformizing $R$. Let $M(R)$ be the open unit ball in $L^{\infty}(R)\left(\cong L^{\infty}(\mathbb{D}, \Gamma)\right)$. Then there is a canonical projection $\Phi: M(R) \rightarrow T(R)$. Namely, $\Phi(\mu)$ is the equivalence class (in $T(R)$ ) of a quasiconformal mapping on $R$ whose complex dilatation is $\mu$. This projection is called the Bers projection.

Let $\mu \in M(\mathbb{D}, \Gamma)$ and let $w^{\mu}: \widehat{\mathbb{C}} \rightarrow \widehat{\mathbb{C}}$ be a quasiconformal mapping fixing $1, i$ and -1 whose dilatation coincides with either $\mu$ on $\mathbb{D}$, or 0 on $\mathbb{D}^{-}=\widehat{\mathbb{C}}-\overline{\mathbb{D}}$. Let $\mathcal{S}(\mu)$ denote the Schwarzian derivative of a conformal mapping $\left.w^{\mu}\right|_{\mathbb{D}^{-}}: \mathbb{D}^{-} \rightarrow \widehat{\mathbb{C}}$. Then it follows from the compatibility of $\mu$ that $\mathcal{S}(\mu) \in B_{2}\left(\mathbb{D}^{-}, \Gamma\right)$. L. Bers showed that there is an embedding $\mathcal{B}_{\Gamma}: T(R) \rightarrow B_{2}\left(\mathbb{D}^{-}, \Gamma\right)$ satisfying $\mathcal{B}_{\Gamma}(\Phi(\mu))=\mathcal{S}(\mu)$ for all $\mu \in M(R)$. This embedding is called the Bers embedding of $T(R)$.

Let $\widehat{M}(R)$ be the unit ball in $\widehat{L}(R)$. In [5], C. Earle, F. Gardiner and N. Lakic proved the existence of a holomorphic splitting submersion $\widehat{\Phi}_{R}: \widehat{M}(R) \rightarrow A T(R)$ and a holomorphic mapping $\widehat{\mathcal{B}}_{\Gamma}: A T(R) \rightarrow \widehat{B}(\Gamma)$ with the following diagram commutative:

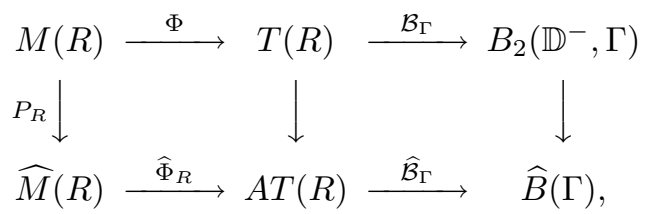

where the vertical directions are canonical projections. It follows from the implicit function theorem that $\widehat{\Phi}_{R}$ admits a local holomorphic section at any point of $A T(R)$ (cf. Theorem 2.11 of [5] and p. 89 of [13]).

In [8, C. Earle, V. Markovic and D. Saric obtained that the mapping $\widehat{\mathcal{B}}_{\Gamma}$ is actually injective and its image is a bounded domain in $\widehat{B}(\Gamma)$. The mapping $\widehat{\mathcal{B}}_{\Gamma}$ is called the asymptotic Bers map from $A T(R)$ to $\widehat{B}(\Gamma)$. 
Lemma 2.1. The image $\widehat{\mathcal{B}}_{\Gamma}(A T(R))$ contains the $1 / 2$-ball with center 0 , and is contained in the $3 / 2$-ball with center 0 in $\widehat{B}(\Gamma)$.

This is derived from Lemma 2.2 below and the fact that the image of the Teichmüller space under the Bers' embedding contains the $1 / 2$-ball with center 0 , and is contained in the $3 / 2$-ball with center 0 (cf. [1, 12, [13).

Lemma 2.2. Let $B=\left(B,\|\cdot\|_{B}\right)$ be a normed space and let $B_{0}$ be a subspace of $B$. Denote by $\pi: B \rightarrow B / B_{0}$ the quotient map. Suppose that a subset $D$ in $B$ contains the $r_{1}$-ball with center 0 and is contained in the $r_{2}$-ball with center 0 . Then $\pi(D)$ satisfies the same conditions with respect to the quotient norm $\|\cdot\|_{B / B_{0}}$ in $B / B_{0}$.

Proof. Since the quotient map is a norm-decreasing map, $\pi(D)$ is contained in the $r_{2}$-ball with center 0 in $B / B_{0}$. Let $[b] \in B / B_{0}$ with $\|[b]\|_{B / B_{0}}<r_{1}$. From the definition of the quotient norm, there is a representative $b \in[b]$ with $\|b\|_{B}<r_{1}$, which implies that $[x] \in \pi(D)$.

\section{INVARIANT PSEUDODISTANCES}

In this section, we give the definition and basic properties of invariant distances on complex Banach manifolds.

3.1. Invariant distances. An invariant distance $d_{X}$ on a complex Banach manifold $X$ is a pseudodistance which satisfies the following inequalities:

$$
d_{\mathbb{D}}\left(f\left(x_{1}\right), f\left(x_{2}\right)\right) \leq d_{X}\left(x_{1}, x_{2}\right) \quad \text { and } \quad d_{X}\left(g\left(z_{1}\right), g\left(z_{2}\right)\right) \leq d_{\mathbb{D}}\left(z_{1}, z_{2}\right)
$$

for every $x_{1}, x_{2} \in X, z_{1}, z_{2} \in \mathbb{D}$, and holomorphic functions $f: X \rightarrow \mathbb{D}$ and $g: \mathbb{D} \rightarrow X$, where $d_{\mathbb{D}}$ is the Poincaré distance on $\mathbb{D}$.

3.2. Inner distances. Let $(X, d)$ be a metric space. A path $\gamma:[a, b] \rightarrow X$ is said to be rectifiable with respect to $d$ if $d$-length

$$
\operatorname{Length}_{d}(\gamma):=\sup \left\{\sum_{k=0}^{m} d\left(\gamma\left(t_{k}\right), \gamma\left(t_{k+1}\right)\right) \mid a=t_{0}<t_{1}<t_{2}<\cdots<t_{m}=b\right\}
$$

of $\gamma$ is finite. We say that $d$ is an inner distance if

$$
d\left(x_{1}, x_{2}\right)=\inf _{\gamma} \operatorname{Length}_{d}(\gamma)
$$

holds for every $x_{1}, x_{2} \in X$, where the infimum is taken over all rectifiable paths connecting $x_{1}$ and $x_{2}$ with respect to $d$. The following proposition follows from the definition.

Proposition 3.1. Let $d_{1}$ and $d_{2}$ be distances on $X$. Suppose that there exist constants $D_{1}$ and $D_{2}$ such that

$$
\frac{1}{D_{1}} d_{1}\left(x_{1}, x_{2}\right) \leq d_{2}\left(x_{1}, x_{2}\right) \leq D_{1} d_{1}\left(x_{1}, x_{2}\right)
$$

for all $x_{1}, x_{2} \in X$ with $d_{1}\left(x_{1}, x_{2}\right) \leq D_{2}$. Then, for any rectifiable path $\gamma$ with respect to $d_{1}$, an inequality

$$
\frac{1}{D_{1}} \text { Length }_{d_{1}}(\gamma) \leq \operatorname{Length}_{d_{2}}(\gamma) \leq D_{1} \operatorname{Length}_{d_{1}}(\gamma)
$$


holds. In particular, if both $d_{1}$ and $d_{2}$ are inner, then

$$
\frac{1}{D_{1}} d_{1}\left(x_{1}, x_{2}\right) \leq d_{2}\left(x_{1}, x_{2}\right) \leq D_{1} d_{1}\left(x_{1}, x_{2}\right)
$$

for all $x_{1}, x_{2} \in X$.

3.3. Carathéodory and Kobayashi distances. Let $X$ be a complex Banach manifold. The Carathéodory pseudodistance $c_{X}$ on $X$ is a pseudodistance defined by

$$
c_{X}\left(x_{1}, x_{2}\right)=\sup \left\{d_{\mathbb{D}}\left(f\left(x_{1}\right), f\left(x_{2}\right)\right) \mid f: X \rightarrow \mathbb{D} \text { holomorphic }\right\}
$$

for $x_{1}, x_{2} \in X$. The Kobayashi pseudodistance $k_{X}$ on $X$ is also a pseudodistance on $X$ which is defined to be

$$
k_{X}\left(x_{1}, x_{2}\right)=\inf \sum_{k=1}^{n} d_{\mathbb{D}}\left(a_{k}, b_{k}\right),
$$

where the infimum is taken over $n \in \mathbb{N}$ and all families of pairs of points $\left\{\left(a_{k}, b_{k}\right)\right\}_{k=1}^{n}$ in $\mathbb{D}$ and holomorphic functions $\left\{f_{k}\right\}_{k=1}^{n}$ with $f_{1}\left(a_{1}\right)=x_{1}, f_{n}\left(b_{n}\right)=x_{2}$, and $f_{k}\left(b_{k}\right)=f_{k+1}\left(a_{k+1}\right)$ for $k=1, \cdots, n-1$. It is known that the kobayashi distance is inner (cf. [9]).

The following properties are well known (cf. [3]).

Lemma 3.1. Let $X$ be a complex Banach manifold. Then:

(a) if $X$ admits a holomorphic embedding into a Banach space whose image is bounded, $c_{X}$ is a distance,

(b) when $X=\mathbb{D}$, both $c_{\mathbb{D}}$ and $k_{\mathbb{D}}$ coincide with the Poincaré distance on $\mathbb{D}$,

(c) let $d_{X}$ be an invariant distance on $X$. Then

$$
c_{X}\left(x_{1}, x_{2}\right) \leq d_{X}\left(x_{1}, x_{2}\right) \leq k_{X}\left(x_{1}, x_{2}\right)
$$

for every $x_{1}, x_{2} \in X$,

(d) $c_{X}$ and $k_{X}$ have the distance-decreasing property, that is,

$$
\begin{aligned}
& c_{X_{2}}\left(F\left(x_{1}\right), F\left(x_{2}\right)\right) \leq c_{X_{1}}\left(x_{1}, x_{2}\right) \quad \text { and } \\
& k_{X_{2}}\left(F\left(x_{1}\right), F\left(x_{2}\right)\right) \leq k_{X_{1}}\left(x_{1}, x_{2}\right)
\end{aligned}
$$

for $x_{1}, x_{2} \in X$ and a holomorphic function $F: X_{1} \rightarrow X_{2}$ between Banach manifolds. Especially, $c_{X}$ and $k_{X}$ are invariant distances, and

(e) if $X$ is the ball with radius $r$ and center the origin in a Banach space $\left(B,\|\cdot\|_{B}\right)$, then any invariant distance on $X$ coincides with the Carathéodory distance and

$$
\|b\|_{B}=r \tanh \left(c_{X}(0, b) / 2\right)=r \tanh \left(k_{X}(0, b) / 2\right)
$$

for $b \in X$.

3.4. A lemma. This section gives a criterion for the completeness of invariant distances on complex Banach manifolds.

Lemma 3.2. Let $X$ be a complex Banach manifold. Suppose that there exist $\delta_{1}, \delta_{2}>0$ and a family of Banach spaces $\left\{B_{x}\right\}_{x \in X}$ such that, for any point $x \in X$, $X$ admits a holomorphic embedding $F_{x}: X \rightarrow B_{x}$ satisfying that $F_{x}(x)=0$ and

$$
\left\{b \in B_{x} \mid\|b\|_{B_{x}}<\delta_{1}\right\} \subset F_{x}(X) \subset\left\{b \in B_{x} \mid\|b\|_{B_{x}}<\delta_{2}\right\},
$$


where $\|\cdot\|_{B_{x}}$ is the norm on $B_{x}$. Then,

(1) $c_{X}$ and $k_{X}$ are locally biLipschitz equivalent with uniform constants, that is, there exist constants $D_{1} \geq 1$ and $D_{2}>0$ depending only on $\delta_{1}$ and $\delta_{2}$ such that

$$
c_{X}(x, y) \leq k_{X}(x, y) \leq D_{1} c_{X}(x, y)
$$

for $x, y \in X$ with $c_{X}(x, y) \leq D_{2}$, and

(2) any invariant distance on $X$ is a complete distance.

Proof. (1) For $x \in X$, we set $U_{x}=F_{x}^{-1}\left(\left\{\|b\|_{B_{x}}<\delta_{1} / 2\right\}\right)$. Then we can see that $c_{X}$ and $k_{X}$ are locally comparable with a uniform constant. Indeed, since $F_{x}$ is a holomorphically embedding with $F_{x}(x)=0$, by (e) in Lemma 3.1.

$$
\tanh ^{-1}\left(\left\|F_{x}(y)\right\|_{B_{x}} / \delta_{2}\right) \leq c_{X}(x, y) \leq k_{X}(x, y) \leq \tanh ^{-1}\left(\left\|F_{x}(y)\right\|_{B_{x}} / \delta_{1}\right)
$$

for $y \in U_{x}$. Since the left-hand side of (3.2) is comparable with the right-hand side on $U_{x}$, and $U_{x}$ contains the $D_{2}$-ball with respect to $c_{X}$ for some $D_{2}=D_{2}\left(\delta_{1}, \delta_{2}\right)>0$ (by (3.2) again), we conclude the assertion.

(2) We only show the case of the Carathéodory distance. The other case is derived from this case because of (b) in Lemma 3.1 and the local comparability in (1).

Let $\left\{x_{i}\right\}_{i=1}^{\infty}$ be a Cauchy sequence with respect to $\left(X, c_{X}\right)$. We may assume that $c_{X}\left(x_{i}, x_{j}\right) \leq 2 \tanh ^{-1}\left(\delta_{1} / 3 \delta_{2}\right)$ for all $i, j \geq 1$. For the sake of simplicity, we set $F=F_{x_{1}}$ and $B=B_{x_{1}}$. Since $F(X) \subset\left\{b \in B \mid\|b\|_{B}<\delta_{2}\right\}$ and $F\left(x_{1}\right)=0$, by (d) and (e) in Lemma 3.1, we have

$$
\left\|F\left(x_{i}\right)\right\|_{B} \leq \delta_{2} \tanh \left(c_{X}\left(x_{1}, x_{j}\right) / 2\right) \leq \delta_{1} / 3 .
$$

This means that $F(X)$ is contained in the ball $\left\{b \in B \mid\left\|b-F\left(x_{i}\right)\right\|_{B} \leq \delta_{2}+\delta_{1} / 3\right\}$ for all $i \geq 1$. Therefore, by (e) in Lemma 3.1 again, we get

$$
\left\|F\left(x_{i}\right)-F\left(x_{j}\right)\right\|_{B} \leq\left(\delta_{2}+\delta_{1} / 3\right) \tanh \left(c_{X}\left(x_{i}, x_{j}\right) / 2\right) .
$$

This implies that $\left\{F\left(x_{i}\right)\right\}_{i=1}^{\infty}$ is a Cauchy sequence with respect to $\left(B,\|\cdot\|_{B}\right)$. Thus, $F\left(x_{i}\right)$ converges to some $b_{\infty} \in B$ with $\left\|b_{\infty}\right\|_{B} \leq \delta_{1} / 3$. Therefore, $b_{\infty} \in F(X)$, and hence, $x_{i}$ converges to $F^{-1}\left(b_{\infty}\right) \in X$.

\section{Proof of the theorems}

We begin the proof of our theorems by stating the following proposition.

Proposition 4.1 (Allowable bijections). Let $R$ and $S$ be Riemann surfaces and let $f$ be a quasiconformal mapping from $R$ to $S$. Then the map $[f]^{*}: A T(S) \rightarrow A T(R)$ defined by $[f]^{*}\left([g]_{A T}\right)=[g \circ f]_{A T}$ is well defined and biholomorphic.

A biholomorphism $[f]^{*}$ in Proposition 4.1 is called an allowable bijection induced by a quasiconformal mapping $f: R \rightarrow S$ (cf. $\S 2.3 .1$ of [13]).

Actually, Proposition 4.1 was already observed by C. Earle, F. Gardiner and N. Lakic in their series of works (cf. [5], 6] and [7]). In their works, the proposition was obtained as a consequence of general principles for holomorphic mappings on domains in Banach spaces. Here, for the convenience of readers, we will try to give a direct proof of Proposition 4.11

Before proving Proposition 4.1 we discuss how Proposition 4.1 is applied to obtain Theorems 1 and 2, Indeed, by Lemma 3.2 it suffices to construct collections

\footnotetext{
${ }^{1}$ In [6], they called $[f]^{*}$ a geometric isomorphism.
} 
of Banach spaces $\left\{B_{x}\right\}_{x \in A T(R)}$ and holomorphic embeddings $\left\{F_{x}\right\}_{x \in A T(R)}$ with $F_{x}: A T(R) \rightarrow B_{x}$ for $x \in A T(R)$ such that $F_{x}(x)=0$ and

$$
\left\{b \in B_{x} \mid\|b\|_{B_{x}}<\delta_{1}\right\} \subset F_{x}(A T(R)) \subset\left\{b \in B_{x} \mid\|b\|_{B_{x}}<\delta_{2}\right\}
$$

for some constants $\delta_{1}$ and $\delta_{2}$ independent of the choice of points in $A T(R)$.

Let $x=[f]_{A T} \in A T(R)$ and let $\Gamma_{f}$ be the Fuchsian group acting on $\mathbb{D}$ which uniformizes $f(R)$. Denote by $\widehat{\mathcal{B}}_{\Gamma_{f}}: A T(f(R)) \rightarrow \widehat{B}\left(\Gamma_{f}\right)$ the asymptotic Bers map as in 92.4 . Then, by Lemma 2.1] and Proposition 4.1], one see that $F_{x}:=\widehat{\mathcal{B}}_{\Gamma_{f}} \circ\left([f]^{*}\right)^{-1}$ and $B_{x}:=\widehat{B}\left(\Gamma_{f}\right)$ satisfies the desired properties.

Proof of Proposition 4.1. We first check the well-definedness and the bijectivity of allowable bijections. Let $g_{1}$ and $g_{2}$ be quasiconformal mappings on $S$ with $\left[g_{1}\right]_{A T}=\left[g_{2}\right]_{A T}$. By definition, there is an asymptotically conformal mapping $h: g_{1}(S) \rightarrow g_{2}(S)$ which is quasiconformally isotopic to $g_{2} \circ g_{1}^{-1} \mathrm{rel} \partial^{i d} g_{1}(S)$. Then $g_{i} \circ f$ is a quasiconformal mapping from $R$ to $g_{i} \circ f(R)=g_{i}(S)$, and $\left(g_{2} \circ f\right) \circ$ $\left(g_{1} \circ f\right)^{-1}=g_{2} \circ g_{1}^{-1}$ is quasiconformally isotopic to an asymptotically conformal mapping $h$ rel $\partial^{i d}\left(g_{1} \circ f(R)\right)$. This means that $\left[g_{1} \circ f\right]_{A T}=\left[g_{2} \circ f\right]_{A T}$ in $A T(R)$. Since $[f]^{*} \circ\left[f^{-1}\right]^{*}=i d_{A T(R)}$ and $\left[f^{-1}\right]^{*} \circ[f]^{*}=i d_{A T(S)},[f]^{*}$ is bijective.

We next show that $[f]^{*}$ is holomorphic. Since the projection $\widehat{\Phi}_{R}: \widehat{M}(R) \rightarrow$ $A T(R)$ is a holomorphic split submersion, this is deduced from the following claim.

Claim 1. $f$ induces a biholomorphic mapping $[f]^{\hat{*}}: \widehat{M}(S) \rightarrow \widehat{M}(R)$ which commutes the following diagram:

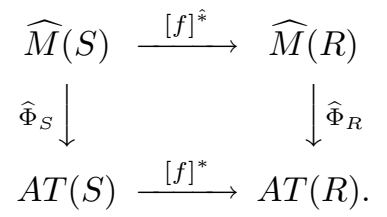

Proof of Claim 1. Consider a biholomorphic mapping $G: M(S) \rightarrow M(R)$ defined by

$$
G(\nu)=\frac{\mu_{f}+f^{*}(\nu)}{1+f^{*}(\nu) \overline{\mu_{f}}}
$$

where $\mu_{f}$ is the complex coefficient of $f$ and $f^{*}(\nu)$ is a Beltrami differential on $R$ defined by the pull-back formula

$$
f^{*}(\nu)=(\nu \circ f) \overline{f_{z}} / f_{z}
$$

By definition, $G(\nu)$ is the complex coefficient of the $\nu$-quasiconformal mapping and $f$. Therefore, $G$ satisfies the following commutative diagram:

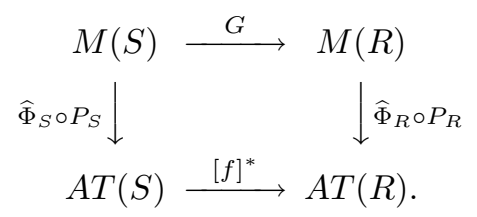

Let $\nu_{1}$ and $\nu_{2}$ be Beltrami coefficients with $\nu_{1}-\nu_{2} \in L_{0}^{\infty}(S)$. Then

$$
G\left(\nu_{1}\right)-G\left(\nu_{2}\right)=\frac{\left(1-\left|\mu_{f}\right|^{2}\right) f^{*}\left(\nu_{1}-\nu_{2}\right)}{\left(1+f^{*}\left(\nu_{1}\right) \overline{\mu_{f}}\right)\left(1+f^{*}\left(\nu_{2}\right) \overline{\mu_{f}}\right)} \in L_{0}^{\infty}(R)
$$


since $f^{*}\left(\nu_{1}-\nu_{2}\right) \in L_{0}^{\infty}(R)$. Therefore, $G$ descends to a bijective mapping $[f]^{*}$ : $\widehat{M}(S) \rightarrow \widehat{M}(R)$. We now check that $[f]^{\hat{*}}$ is holomorphic. The continuity follows from (4.1) since $\left\|f^{*}\left(\nu_{i}\right)\right\|_{\infty}=\left\|\nu_{i}\right\|_{\infty}<1$ and

$$
\left\|[f]^{\hat{*}}\left(\left[\nu_{1}\right]_{\hat{L}}\right)-[f]^{\hat{*}}\left(\left[\nu_{2}\right]_{\hat{L}}\right)\right\|_{\hat{L}} \leq \frac{1}{\left(1-\left\|\mu_{f}\right\|_{\infty}\right)^{2}}\left\|\left[\nu_{1}\right]_{\hat{L}}-\left[\nu_{1}\right]_{\hat{L}}\right\|_{\hat{L}}
$$

Let $\nu_{1}, \nu_{2} \in M(S)$ and $\tau_{1}, \tau_{2} \in L^{\infty}(S)$ with $\nu_{1}-\nu_{2}, \tau_{1}-\tau_{2} \in L_{0}^{\infty}(S)$. By (4.1), we have

$$
\frac{1}{\epsilon}\left(G\left(\nu_{i}+\epsilon \tau_{i}\right)-G\left(\nu_{i}\right)\right)=\frac{\left(1-\left|\mu_{f}\right|^{2}\right) f^{*}\left(\tau_{i}\right)}{\left(1+f^{*}\left(\nu_{i}\right) \overline{\mu_{f}}\right)\left(1+f^{*}\left(\nu_{i}+\epsilon \tau_{i}\right) \overline{\mu_{f}}\right)}
$$

for $i=1,2$. Hence,

$$
\begin{aligned}
\lim _{\epsilon \rightarrow 0} \frac{1}{\epsilon}\left(G\left(\nu_{1}+\epsilon \tau_{1}\right)-G\left(\nu_{1}\right)\right)-\lim _{\epsilon \rightarrow 0} \frac{1}{\epsilon}\left(G\left(\nu_{2}+\epsilon \tau_{2}\right)-G\left(\nu_{2}\right)\right) \\
\quad=\frac{\left(1-\left|\mu_{f}\right|^{2}\right) f^{*}\left(\tau_{1}\right)}{\left(1+f^{*}\left(\nu_{1}\right) \overline{\mu_{f}}\right)^{2}}-\frac{\left(1-\left|\mu_{f}\right|^{2}\right) f^{*}\left(\tau_{2}\right)}{\left(1+f^{*}\left(\nu_{2}\right) \overline{\mu_{f}}\right)^{2}}=\frac{\left(1-\left|\mu_{f}\right|^{2}\right) \mathcal{M}}{\left(1+f^{*}\left(\nu_{1}\right) \overline{\mu_{f}}\right)^{2}\left(1+f^{*}\left(\nu_{2}\right) \overline{\mu_{f}}\right)^{2}},
\end{aligned}
$$

where

$$
\begin{aligned}
& \mathcal{M}= f^{*}\left(\tau_{1}\right)-f^{*}\left(\tau_{2}\right)+2\left(f^{*}\left(\tau_{2}\right) f^{*}\left(\nu_{1}\right)-f^{*}\left(\tau_{1}\right) f^{*}\left(\nu_{2}\right)\right) \overline{\mu_{f}} \\
& \quad+\left(f^{*}\left(\tau_{2}\right) f^{*}\left(\nu_{1}\right)^{2}-f^{*}\left(\tau_{1}\right) f^{*}\left(\nu_{2}\right)^{2}\right) \overline{\mu f}^{2} \\
&=\left\{f^{*}\left(\tau_{1}\right)-f^{*}\left(\tau_{2}\right)\right\} \quad+2\left\{\left(f^{*}\left(\tau_{2}\right)-f^{*}\left(\tau_{1}\right)\right) f^{*}\left(\nu_{1}\right)+f^{*}\left(\tau_{1}\right)\left(f^{*}\left(\nu_{1}\right)-f^{*}\left(\nu_{2}\right)\right)\right\}{\overline{\mu_{f}}}^{*} \\
& \quad \quad+\left\{f^{*}\left(\tau_{2}\right)\left(f^{*}\left(\nu_{1}\right)^{2}-f^{*}\left(\nu_{2}\right)^{2}\right)+\left(f^{*}\left(\tau_{2}\right)-f^{*}\left(\tau_{1}\right)\right) f^{*}\left(\nu_{2}\right)^{2}\right\}{\overline{\mu_{f}}}^{2} \\
& \quad \\
& \quad L_{0}^{\infty}(R) .
\end{aligned}
$$

Therefore, for $p=[\nu]_{\hat{L}} \in \widehat{M}(S)$ and $v=[\tau]_{\hat{L}} \in \widehat{L}(S)=T_{p} \widehat{M}(S)$, the complex Gateaux derivative

$$
\lim _{\epsilon \rightarrow 0} \frac{1}{\epsilon}\left([f]^{\hat{*}}(p+\epsilon v)-[f]^{\hat{*}}(p)\right)
$$

exists and is equal to

$$
\left[\lim _{\epsilon \rightarrow 0} \frac{1}{\epsilon}(G(\nu+\epsilon \tau)-G(\nu))\right]_{\hat{L}}=\left[\frac{\left(1-\left|\mu_{f}\right|^{2}\right)}{\left(1+f^{*}(\nu) \overline{\mu_{f}}\right)^{2}} f^{*}(\tau)\right]_{\hat{L}},
$$

which leads what we desired (cf. $\S 1.6 .1$ of [13]).

The following corollaries follow from Proposition 4.1

Corollary 3. The complex structure on $A T(R)$ is canonical in the sense that it is independent of the choice of the base surface. Namely, $A T(R)$ and $A T(S)$ are biholomorphic when $R$ and $S$ are quasiconformally equivalent.

Corollary 4. Aut $(A T(\mathbb{D}))$ acts transitively on $A T(\mathbb{D})$.

\section{ACKNOWLEDGEMENT}

The author expresses his hearty gratitude to Professor Clifford J. Earle for his many valuable suggestions and comments and for informing the proof of Proposition 4.1 given by him and his collaborators. He also thanks the referee of the previous version of this paper for useful comments. 


\section{REFERENCES}

[1] Ahlfors L.V. and Weill G., A uniqueness theorem for Beltrami equations, Proc. Amer. Math. Soc. 13 (1962), 385-404. MR0148896 (26:6393)

[2] Ahlfors L.V. and Sario L., Riemann surfaces, Princeton University Press (1960). MR0114911 $(22: 5729)$

[3] Dineen S., The Schwarz Lemma, Oxford Mathematical Monographs, Clarendon Press (1989). MR:1033739 (91f:46064)

[4] Earle C., On the Carathéodory metric in Teichmüller spaces, Discontinuous groups and Riemann surfaces (Proc. Conf., Univ. Maryland, College Park, Md., 1973), Ann. of Math. Studies 79, Princeton Univ. Press (1974), 99-103. MR0352450 (50:4937)

[5] Earle C.J., Gardiner F. and Lakic N., Asymptotic Teichmüller space, Part I: The complex structure, Comtemp. Math. 256 (2000), 17-38. MR.1759668 (2001m:32029)

[6] Earle C.J., Gardiner F. and Lakic N., Asymptotic Teichmüller space, Part II: The metric structure, Contemporary Math. 355 (2004), 187-219. MR1759668 (2001m:32029)

[7] Earle C.J., Gardiner F. and Lakic N., Finsler structures and their quotients on domains in Banach spaces, Complex Dynamics and Related Topics, IP, New Studies in Math 5 (2004), 30-49.

[8] Earle C.J., Markovic J. and Saric D., Barycentric extension and the Bers embedding for asymptotic Teichmüller space, Comtemp. Math. 311 (2002), 85-105. MR1940165(2003i:30072)

[9] Franzoni T. and Vesentini E., Holomorphic maps and Invariant distances, Notas de Matemática, 69. North-Holland Publishing Co., Amsterdam, New York (1980). MR0563329 (82a:32032)

[10] Gardiner F., The Teichmüller-Kobayashi metric for infinite-dimensional complex Teichmüller spaces. Kleinian groups and related topics (Oaxtepec, 1981), Lecture Notes in Math. 971, Springer, Berlin, New York (1983), pp. 48-67. MR0690278 (84f:32025)

[11] Gardiner F. and Lakic N., Quasiconformal Teichmüller theory, Mathematical Surveys and Monographs 76, American Mathematical Society (2000). MR.1730906 (2001d:32016)

[12] Imayoshi Y. and Taniguchi M., An introduction to Teichmüller spaces, Springer-Verlag, Tokyo (1992). MR1215481 (94b:32031)

[13] Nag S., The Complex Analytic Theory of Teichmüller spaces, Wiley-Interscience, New York (1998). MR0927291 (89f:32040)

Department of Mathematical Sciences, Tokyo Denki University, Ishizaka, Hatoyama, Hiki SAITAMA, 359-0394, JAPAN

E-mail address: miyachi@r.dendai.ac.jp 